\title{
REVISIÓN DE LOS RESTOS ÓSEOS HUMANOS DEL SITIO LA PETRONA (TRANSICIÓN PAMPEANO-PATAGÓNICA ORIENTAL, ARGENTINA)
}

\author{
GUSTAVO FLENSBORG*, GUSTAVO MARTÍNEZ", MARIELA GONZÁLEZ" Y PABLO BAYALA*
}

\begin{abstract}
RESUMEN
La arqueología del curso inferior del río Colorado (transición Pampeano-Patagónica Oriental) contó con su primer trabajo sistemático a partir de la excavación del sitio La Petrona (sur de la provincia de Buenos Aires). El registro óseo humano recuperado del sitio fue objeto de un análisis bioarqueológico que es retomado en este trabajo. En este sentido, se re-analizan las modalidades de entierro, la frecuencia de las unidades anatómicas representadas, el NMI, el sexo y edad de los mismos, las paleopatologías y la tafonomía. Los resultados de estas vías de análisis permitieron lograr un nuevo estado de la cuestión para algunas de estas variables, redefinir la cronología del sitio (Holoceno tardío final) y desarrollar un escenario que permite abordar temas referidos a las prácticas de inhumación, a la complejidad en el tratamiento de los muertos y su vinculación con el uso recurrente de ciertos sectores del paisaje.
\end{abstract}

PALABRAS CLAVE: bioarqueología, tafonomía, prácticas mortuorias, Holoceno tardío final.

\section{A REVIEW OF THE HUMAN BONE REMAINS FROM LA PETRONA SITE (EASTERN PAMPEAN-PATAGONIAN TRANSITION, ARGENTINA)}

\section{ABSTRACT}

The first systematic archaeological work carried out in the lower basin of the Colorado River (Eastern Pampean-Patagonian transition, southern Buenos Aires province) was the excavation of La Petrona site. The most outstanding findings were the primary and secondary burials, which were previously published (Martínez and Figuerero Torres, 2000). In this paper, a re-analyses of the human bones that make up these burials is carried out. Burial modalities, frequency of represented skeletal parts, MNI that compose the burials, sex and age, paleopathologies, as well as taphonomic information are presented. As a 
result of these analyses, new information about the site emerges leading to a revision of the chronology (Final Late Holocene), and to the discussion of issues such as inhumation practices, complex scenarios in corpse treatment and manipulation, and their relationship with the recurrent use of places throughout the landscape.

KEY WORDS: bioarchaeology, taphonomy, mortuary practices, Final Late Holocene.

\section{INTRODUCCIÓN}

La Petrona (Pdo. de Villarino, Pcia. de Buenos Aires) fue el primer sitio arqueológico estudiado sistemáticamente en el curso inferior del río Colorado y sentó las bases para el desarrollo posterior de un proyecto de investigación que se viene llevando a cabo desde hace una década (Martínez 20082009). En la primera contribución sobre este sitio, Martínez y Figuerero Torres (2000) realizaron un exhaustivo estado de la cuestión respecto de los entierros humanos para el sector de transición Pampeano-Patagónico Oriental y presentaron los primeros resultados sobre modalidades de entierro (e.g., primarias, secundarias, simples y múltiples), partes esqueletarias representadas, sexo y edad de los individuos, paleopatologías y tafonomía.

Con el desarrollo del proyecto antes mencionado los estudios bioarqueológicos han experimentado un crecimiento importante (Bayala 2010; Bayón et al. 2004; Flensborg 2010; González 2010; Martínez et al. 2007; Prates et al. 2006). En este sentido, no solo se generó información para el área sino que surgieron nuevas preguntas y se estandarizó el empleo de metodologías de análisis en los estudios. En este marco, el objetivo del trabajo es re-analizar los entierros humanos del sitio La Petrona con el propósito de abordar con mayor profundidad temas ya tratados y otros novedosos. Se analizan las modalidades de inhumación, la cronología de los eventos de muerte y de los entierros, las historias tafonómicas de los mismos, el NMI que componen los entierros secundarios, el sexo, la edad y la salud de los individuos inhumados. Esta información puesta en conjunción, permitirá discutir temas como complejidad de prácticas mortuorias, el uso recurrente del paisaje (posiblemente gatillado por éstas prácticas), el manejo de los cuerpos y su temporalidad.

\section{BREVE CARACTERIZACIÓN DEL SITIO}

En este apartado se brindan las principales características del sitio, aunque información detallada sobre el mismo puede consultarse en Martínez y Figuerero Torres (2000). El sitio La Petrona (39 $30^{\prime}$ 13.92" LS y 62 47 '07.99" LO) se encuentra sobre un médano destacado del paisaje por sus dimensiones (ca. 90 x $30 \mathrm{~m}$ ) y localizado a ca. $200 \mathrm{~m}$ de la margen norte del río Colorado (Fig. 1). El mismo fue desmontado en 1985 y sujeto a erosión, procesos que produjeron la exposición parcial de algunos de los entierros en el año 1988. En ese mismo año y en 1991 se realizaron las tareas de recuperación de los entierros y la excavación de seis sondeos en derredor de los mismos (Fig. 1). En el año 2000 se llevaron a cabo nueve sondeos en los cuales se obtuvo muy escasa cantidad de materiales arqueológicos y no se detectaron nuevos entierros. Estos resultados, sumados al registro principalmente superficial de artefactos (e.g., líticos, cerámicos, etc.), permiten reforzar la idea de que buena parte del médano $(\mathrm{ca} .70 \mathrm{~cm})$ fue deflacionado y que aquello que se encontraba en estratigrafía se localiza hoy en la superficie actual del médano, formando un palimpsesto (ver Martínez y Figuerero Torres 2000). Este conjunto artefactual integrado por numerosas puntas de proyectil, materiales de molienda, cerámica, adornos labiales y/o auriculares, material faunístico muy fragmentado y escaso material histórico (e.g., botón de uniforme, cartuchos de explosivos actuales, etc.) ya ha sido descrito e informado en trabajos previos (Martínez 2008-2009; Martínez y Figuerero Torres 2000).

Dentro de la superficie del médano antes citada, cuatro entierros humanos fueron hallados en un área de ca. $36 \mathrm{~m}^{2}$. Dos de ellos son secundarios múltiples (LP1 y LP2) y se encuentran muy próximos entre sí, mientras que los restantes (LP3 y LP4) son primarios simples. Siete fechados radiocarbónicos los ubican en el Holoceno tardío Final (ca. 770-250 años AP; véase Tabla 4 en Martínez et al. 2009). 


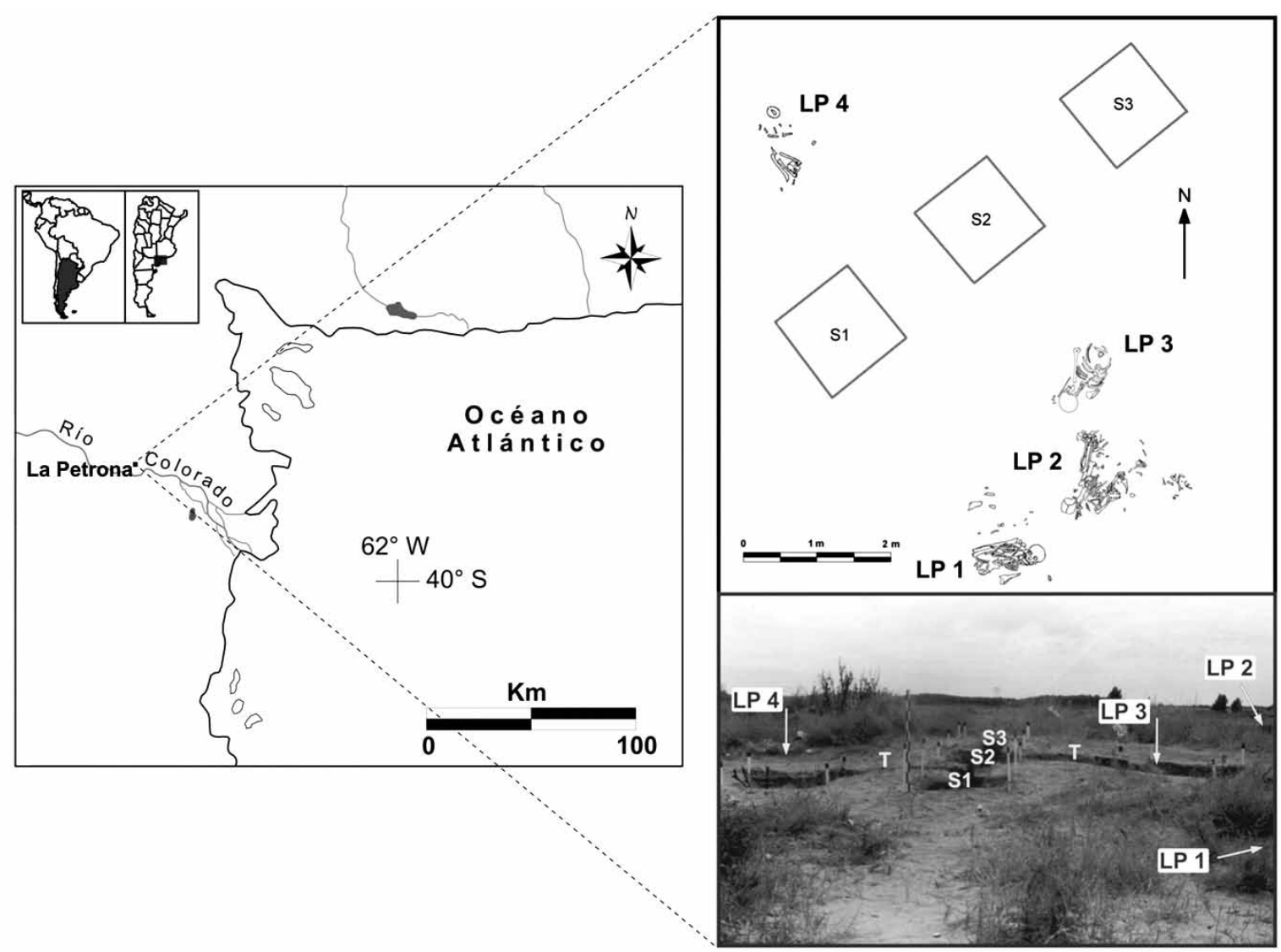

Fig. 1. Ubicación geográfica del sitio La Petrona. Esquema de distribución de los entierros en el médano y ubicación de tres sondeos y dos trincheras. Nota: $\mathrm{T}=$ trincheras; $\mathrm{S}=$ sondeo.

Las características principales de estos entierros son discutidas mas abajo.

\section{METODOLOGÍA}

El análisis del registro bioarqueológico involucró determinaciones anatómicas, de lateralidad y el cálculo de índices cuantitativos básicos tales como el número mínimo de individuos (NMI) y el número mínimo de elementos (NME) (Lyman 1994; Reitz y Wing 1999). Para aquellos ítems con una mala preservación se procedió al remontaje de los fragmentos con la finalidad de lograr una mayor integridad de las piezas óseas que permitan realizar las determinaciones arriba mencionadas.

En cuanto a la estimación de la edad de muerte, se efectuaron observaciones morfoscópicas y mediciones osteométricas. En los individuos suba- dultos, se analizaron las dimensiones de los huesos y el estado de fusión de sus epífisis (Scheuer y Black 2000); mientras que en los individuos adultos, se registraron los cambios en la superficie articular de la sínfisis púbica (Brooks y Suchey 1990; Gilbert y McKern 1973; Suchey y Kats 1986) y los cambios morfológicos en la superficie auricular del ilion (Lovejoy et al. 1985). Simultáneamente, se consideraron algunos marcadores secundarios que, si bien tienen un carácter relativo y son escasamente utilizados, conforman una herramienta útil para la estimación de esta variable. En este caso, se evaluó la fusión de ciertas epífisis y/o segmentos óseos durante la adultez temprana (e.g., sacro, epífisis de la clavícula y extremo vertebral de las costillas; Buikstra y Ubelaker 1994).

La determinación del sexo se llevó a cabo mediante observaciones macroscópicas sobre dife- 
rentes indicadores morfológicos. En cuanto al cráneo, se consideró el desarrollo de la cresta nucal y de la glabela, el aspecto de la margen supraorbital y el tamaño del proceso mastoideo, mientras que para la mandíbula se empleó el desarrollo de la eminencia mentoniana. En cuanto a los coxales, se analizó la escotadura ciática mayor, el surco preauricular, el arco en la faceta auricular del ilion, el aspecto medial de la rama isquiopúbica, el arco ventral y la concavidad o ángulo subpúbico. Respecto del sacro, se relevó el ancho y el largo del hueso, la curvatura longitudinal y la extensión de la carilla auricular (Bass 1995; Buikstra y Ubelaker 1994).

Con el fin de identificar los procesos naturales y culturales que actuaron sobre los restos óseos humanos, se llevó a cabo un análisis tafonómico analizando el tejido cortical a ojo desnudo, con lupa unifocal de 20X y con estereomicroscopio de 40X. En este análisis se dio suma importancia a las huellas conspicuas e inconspicuas, y se consideró además el potencial de identificabilidad que se puede obtener sin la necesidad de altos aumentos (Blumenschine et al. 1996). Los efectos tafonómicos que se tuvieron en cuenta fueron meteorización, integridad, fracturas, pérdida ósea, acción de roedores y carnívoros, marcas de raíces y huellas antrópicas intencionales ((Behrensmeyer 1978; Binford 1981; Bocek 1986; Fisher 1995; Lyman 1994).

A nivel paleopatológico se llevó a cabo un análisis macroscópico y con lupa binocular (40X). Se tuvo en cuenta la localización y extensión de las lesiones (medida en milímetros con un calibre digital) en cada elemento óseo y, cuando fue posible, la distribución de las anomalías en un mismo individuo. Se evaluaron diferentes grupos etiológicos: a) procesos degenerativos (Jurmain 1991; Weiss 2006), b) traumáticos (Aufderheide y Rodríguez-Martín 1998; Ortner 2003) y c) infecciosos (Ortner 2003; Powell 1988).

\section{PRINCIPALES CARACTERÍSTICAS DE LOS ENTIERROS}

\section{Entierro 1 (LP1)}

Se trata de un entierro secundario múltiple. Los análisis permitieron contabilizar un total de 96 elementos óseos y un número mínimo de 3 individuos (Tabla 1). La información obtenida respecto al sexo y edad de muerte indica que se hallan representados dos individuos femeninos adultos (35-45 años de edad) y un individuo adulto de sexo indeterminado (Tabla 1). El único cráneo recuperado en el entierro presenta deformación tabular erecta, variedad planolambdica (Martínez y Figuerero Torres 2000). Dos fechados radiocarbónicos indican una cronología de $352 \pm 51$ años AP y $314 \pm 45$ años AP (Martínez 2004).

\section{Entierro 2 (LP2)}

Se trata de un entierro secundario múltiple. Los análisis permitieron calcular un total de 124 elementos óseos, representando un número mínimo de 3 individuos (Tabla 1). La mayoría de las unidades anatómicas recuperadas ( $\mathrm{n}=99$ ) corresponden a dos individuos adultos, uno de ellos femenino (35-45 años de edad) y el restante de sexo indeterminado. Asimismo, se registró la presencia de 25 huesos correspondientes a manos y pies, de tamaño y morfología pequeña, sin fusionar, asignables a la categoría subadulto (Tabla 1$)$, que indica la presencia de un individuo infante (0-3 años de edad). El único cráneo recuperado muestra una deformación de tipo tabular erecta, variedad planolambdica (Martínez y Figuerero Torres 2000). Dos fechados radiocarbónicos sitúan al entierro entre $481 \pm 37$ años AP y $770 \pm 49$ años AP (Martínez 2004).

\section{Entierro 3 (LP3)}

Se trata de un entierro primario simple incompleto. Los análisis permitieron calcular un total de 92 elementos óseos, correspondientes a un individuo femenino adulto (25-35 años de edad) (Tabla 1). Un rasgo distintivo de este entierro es la ausencia de las últimas vértebras lumbares, coxales, huesos largos de los miembros inferiores, esternón y clavículas. El cráneo no presenta deformación cultural (Martínez y Figuerero Torres 2000). Dos fechados radiocarbónicos arrojaron edades de 411 \pm 39 años AP y $462 \pm 39$ años AP (Martínez 2004).

\section{Entierro 4 (LP4)}

Se trata de un entierro primario simple incompleto. Los análisis cuantitativos señalan la presencia de 42 elementos óseos, correspondientes a un individuo femenino adulto (35-45 años de edad) (Tabla 1). Las unidades anatómicas, a diferencia de las otras inhumaciones, se hallaron dispersas en el contexto de hallazgo y poco articuladas. El cráneo 
Tabla 1. Cuantificación y caracterización de los entierros del sitio La Petrona.

\begin{tabular}{|c|c|c|c|c|c|c|c|c|}
\hline \multirow{2}{*}{ Entierro } & \multirow{2}{*}{ Modalidad de inhumación } & \multirow{2}{*}{ NME } & \multirow{2}{*}{ NMI } & \multicolumn{3}{|c|}{ Sexo } & \multicolumn{2}{|c|}{ Edad } \\
\hline & & & & $\mathrm{M}$ & $\mathrm{F}$ & I & Adulto & Subadulto \\
\hline LP1 & Secundario múltiple & 96 & 3 & - & 2 & 1 & 3 & - \\
\hline LP2 & Secundario múltiple & 124 & 3 & - & 2 & 1 & 2 & 1 \\
\hline LP3 & Primario simple & 92 & 1 & - & 1 & - & 1 & - \\
\hline LP4 & Primario simple & 42 & 1 & - & 1 & - & 1 & - \\
\hline & Total & 354 & 8 & - & 6 & 2 & 7 & 1 \\
\hline
\end{tabular}

presenta deformación tabular erecta, variedad planolambdica (Martínez y Figuerero Torres 2000). Un fechado radiocarbónico indica una cronología de $248 \pm 39$ años AP (Martínez 2008-2009).

\section{ANÁLISIS TAFONÓMICO}

Se presentarán a continuación las tendencias generales obtenidas a partir del análisis tafonómico y se hará especial énfasis en los resultados vinculados a las huellas antrópicas intencionales. De todas las variables analizadas, las que han tenido mayor incidencia son aquellas que implicaron la pérdida de tejido óseo. En el caso del entierro LP1, el 67\% de las unidades óseas están fracturadas, siendo de origen postdepositacional y reciente, con un leve predominio del primero (Tabla 2). Las fracturas postdepositacionales hacen referencia a aquellas que se produjeron mientras los huesos permanecieron enterrados, en tanto que las recientes fueron generadas durante y a posteriori de la recuperación de los entierros. Además, el 100\% de los elementos tienen pérdidas pequeñas y extensivas de tejido óseo producidas en ambas instancias. Con respecto al estado de la superficie de los huesos, el 57\% presenta líneas y estrías de desecación. En este entierro, el $44 \%$ de los huesos se encuentran dentro del rango de integridad anatómica más elevada (76-100\%). En LP2, el 35\% de las unidades óseas presentan fracturas recientes y postdepositacionales en cantidades similares. Las pérdidas de tejido ocurrieron en la mayoría de las piezas (95\%), predominando las postdepositacionales (Tabla 2). Casi la totalidad de los elementos (88\%) que componen este entierro han sido modificados superficialmente por la acción de las raíces. También aquí las líneas de desecación han alterado los huesos, aunque en baja frecuencia (24\%). El 60\% de los elementos poseen una integridad alta, cayendo en el rango de representación ósea de 76-100\%. Las unidades anatómicas correspondientes al individuo infante se encuentran dentro de los rangos más altos de integridad. No obstante, existieron pérdidas del tejido óseo en todos los elementos. En el entierro LP3, sólo el 21\% de las piezas esqueletarias presentan fracturas, con cantidades equiparables para ambos tipos (Tabla 2). El 86\% de las unidades anatómicas han perdido tejido óseo en porciones pequeñas a moderadas y, mayoritariamente, durante el transcurso de su historia postdepositacional. Todos los huesos de este individuo se hallan afectados por las raíces siendo, en general, abundantes y moderadas las cantidades de marcas por elemento. En este caso, muy pocas unidades esqueletarias fueron afectadas por la desecación (37\%). El 87\% de las unidades anatómicas se encuentran dentro del rango de integridad más elevado (76-100\%). Finalmente, el entierro LP4 posee un 38\% de elementos óseos con fracturas postdepositacionales y recientes, en porcentajes similares (Tabla 2). Las pérdidas de tejido óseo ocurrieron en todos los elementos presentes, predominando las postdepositacionales. Respecto de las marcas de raíces, es baja la frecuencia de piezas afectadas (33\%) y es escasa la cantidad de trazas por unidad. Asimismo, el ca. 67\% de los elementos han mantenido una integridad macroestructural alta, ubicándose dentro de los rangos de 51-75\% y $76-100 \%$.

Los elementos que componen los cuatro entierros presentan similares características en cuanto a las alteraciones sufridas luego de su depositación. Su estado general de preservación es regular debido a los procesos diagenéticos que actuaron en los huesos generando friabilidad, líneas de desecación y grietas en la macroestructura ósea. Asimismo, las raíces fueron otra fuente principal de alteración, provocando modificaciones tanto químicas (e.g., disolución) como mecánicas (e.g., improntas) en las unidades óseas. Cabe destacar la ausencia de meteorización y de marcas de carnívoros y roedores en todos los entierros (Tabla 2). 
Tabla 2. Distribución de frecuencias de las variables tafonómicas.

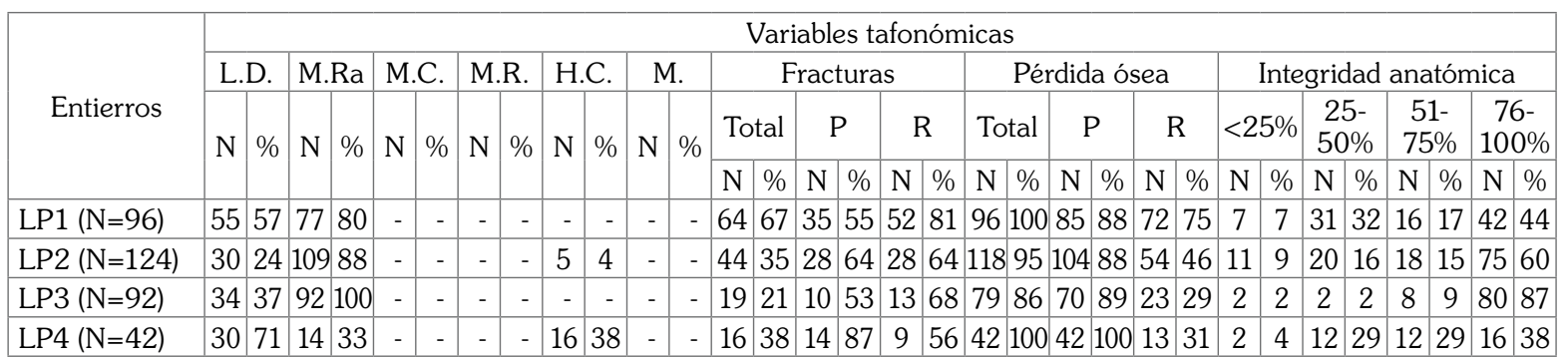

Nota: L.D.= líneas, estrías y grietas de desecación; M.Ra= marcas de raíces; M.C.= marcas de carnívoros; M.R.= marcas de roedores; H.C.= huellas de corte (involucra todas las categorías de procesamiento identificadas); $\mathrm{M}$ = meteorización; $\mathrm{P} .=$ postdepositacionales; $\mathrm{R} .=$ recientes.

Respecto de las evidencias de procesamiento de los cuerpos enterrados en el sitio, se registraron una serie de huellas de origen antrópico en dos de los entierros y con distintas frecuencias de elementos óseos afectados (para una discusión de la aplicación de categorías como huellas de corte en el registro óseo humano véase González 2010). En LP2, se observaron huellas de procesamiento antrópico (4\%) vinculados con actividades de raspado (57\%) y de corte (43\%) (Fig. 2A y 2B; Tabla 2). Cabe mencionar que éstas son pequeñas y de una visibilidad reducida, en la mayor parte de los casos, por la acción de raíces y por la presencia de líneas de desecación. Estas características sumadas a las mencionadas anteriormente respecto del estado general de las unidades anatómicas, apoyarían la hipótesis de que más elementos que los registrados pudieron haber sido alterados por las distintas acciones humanas vinculadas al procesamiento de los cuerpos. Se propone que diversos procesos postdepositacionales habrían enmascarado las huellas culturales (véase González 2010), siendo la acción de la raíces una de las causas principales.
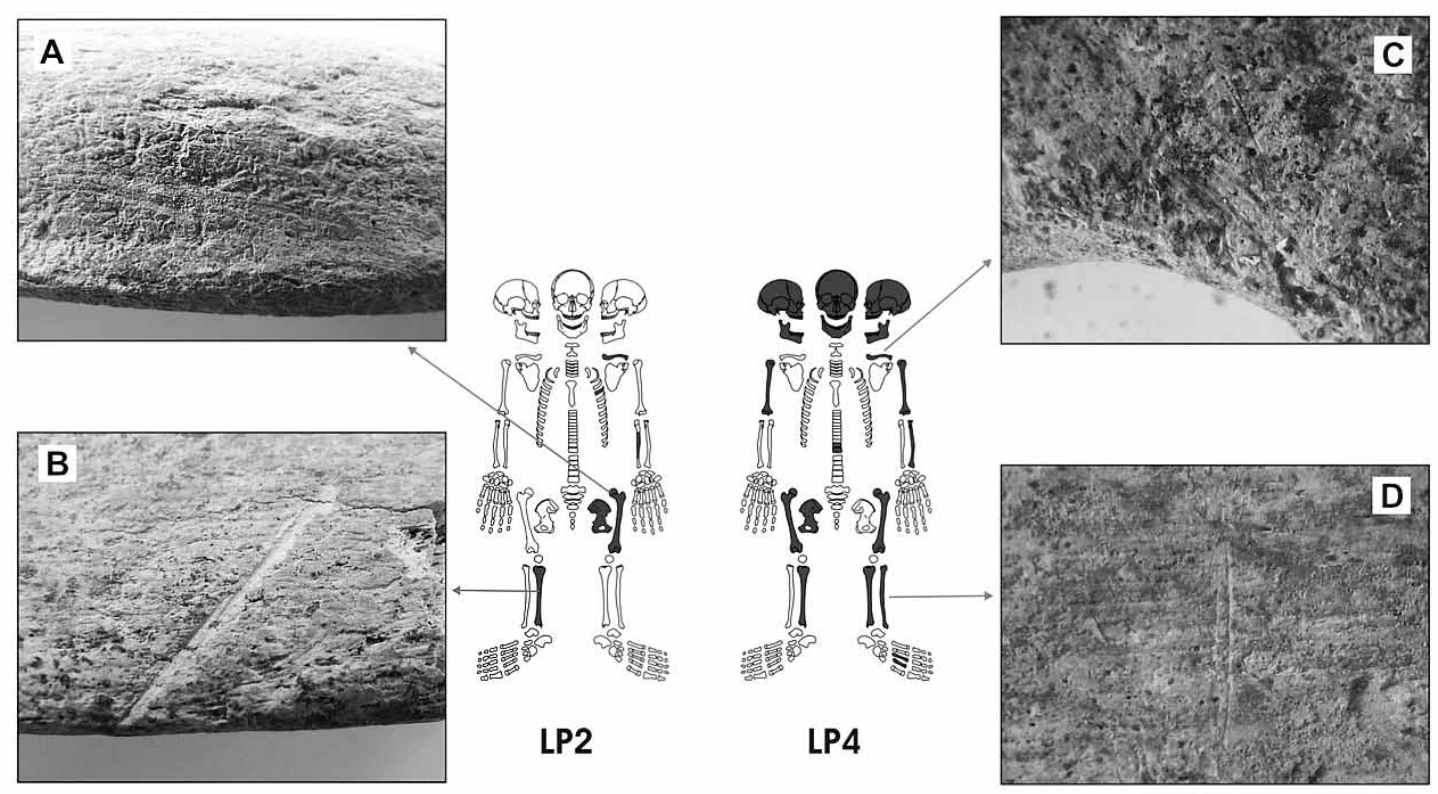

Fig. 2. Los elementos óseos pintados en gris son los que presentan huellas de procesamiento antrópico. Detalle de algunas trazas: A) huella de raspado sobre diáfisis media de fémur izquierdo; B) huella de corte sobre diáfisis distal de tibia derecha; C) huella de raspado sobre porción acromial de clavícula izquierda; D) huella de corte sobre diáfisis media de peroné izquierdo. 
El entierro LP4 también muestra claras trazas de la acción humana sobre el cuerpo del difunto. En este caso, la frecuencia de elementos modificados es mayor que en LP2 así como lo es también la cantidad de huellas observadas. De las 42 unidades anatómicas recuperadas, 16 (ca. 38\%) muestran huellas de procesamiento, de las cuales 6 (ca. 38\%) poseen huellas vinculadas con actividades de descarne, mientras que la totalidad de los elementos (100\%) presentan evidencias de raspado (Fig. 2C y 2D). A diferencia de LP2, las huellas registradas tienen buena visibilidad y, en algunos casos, se hallan agrupadas. Este patrón respondería a que la mayoría de los huesos afectados caen dentro de los rangos de integridad más altos (51-75\% y 76-100\%) y que la frecuencia de elementos afectados por raíces es la más baja.

\section{ANÁLISIS PALEOPATOLÓGICO}

En todos los huesos recuperados de LP1 se registraron 42 lesiones óseas (44\%), correspondiendo el $98 \%$ de las mismas a procesos degenerativos y el $2 \%$ restante a traumáticos. En el caso de los primeros, si se toma en cuenta el número de anomalías patológicas en relación con el total de huesos recuperados para cada porción del esqueleto, se observa que la axial se encuentra más afectada (67\%) que la apendicular (37\%) (Tabla 3). Los huesos con mayor presencia de anomalías de índole degenerativa fueron las vértebras. Las mismas mostraron modificaciones óseas que incluyeron el desgaste del cuerpo vertebral y de las facetas articulares, y el desarrollo de osteofitosis marginal (Fig. 3A). Asimismo, los huesos de las cinturas mostraron labiación marginal y osteocondritis (Fig. 3B). En lo que respecta a los procesos traumáticos, se registró un trauma por aplastamiento en la porción media-anterior de una tibia izquierda, caracterizada por su pequeña dimensión y su forma elongada en dirección al eje sagital del hueso.

En LP2, en el individuo infante no se registró ningún tipo de lesión. En cambio, en los dos adultos el $42 \%$ de los huesos presentaron algún indicador paleopatológico. De las 42 lesiones, el 95\% fueron degenerativas y el $5 \%$ traumáticas. Como se observa en la Tabla 3, el número de huesos con patologías degenerativas sobre la muestra total para cada sector esqueletal, indica que el 83\% afectó al axial y el 27\% al apendicular. Como en el caso anterior, las vértebras, principalmente las últimas cervicales, dorsales y lumbares, junto con el sacro son los elementos óseos más alterados (Fig. 3C). En el caso de las cinturas, se destaca la presencia de forámenes en los sectores laterales de las articulaciones de un coxal izquierdo y de una escápula derecha (Fig. 3D). Se registraron escasas alteraciones en los huesos de la mano (e.g., falanges) y del pie (e.g., tarsos y metatarsos), rasgos que no se habían observado en LP1. En lo que respecta a los traumas, se registró una anquilosis entre la porción distal de una primera falange y la epífisis proximal de la segunda falange de la mano, en estado totalmente remodelado. Esta fusión se dio de manera oblicua, en un ángulo de $45^{\circ}$ hacia la cara ventral. Asimismo, se relevó enteroexostosis en la diáfisis de un quinto metacarpo.

En LP3, el 55\% de los huesos se encontraron alterados por lesiones óseas. De este total, el 96\% corresponde a procesos degenerativos y el $4 \%$ restante a traumáticos. En el caso de los primeros, si se tiene en cuenta el número de lesiones en relación al total de huesos cuantificados, se observó una distribución semejante para ambos sectores del esqueleto (Tabla 3). En este entierro, las vértebras presentaron osteofitosis marginal en los cuerpos y en las facetas articulares, principalmente en las últimas dorsales y lumbares. Similares lesiones fueron observadas en las epífisis de los huesos largos de los miembros superiores, escápulas y huesos de las manos y pies. A nivel traumático, se registró leve miositis osificante asociada a enteroexostosis en un primer y un cuarto metacarpo derechos. Asimismo, en el cráneo se observó una erosión ovoide de escasa profundidad, en la porción medio-derecha del frontal.

En LP4 se registró un 31\% de lesiones óseas. De este porcentaje, el $46 \%$ corresponde a procesos infecciosos, el $8 \%$ a traumáticos y el $46 \%$ a degenerativos (Tabla 3). Para este último indicador, los huesos más afectados fueron las vértebras lumbares y la primera falange de la mano. En este individuo se relevaron lesiones óseas de carácter infeccioso sistémico que afectaron a los huesos de los miembros inferiores. En este sentido, se registró periostitis en la porción tercio-proximal del cuarto metatarso izquierdo y sobre las diáfisis de la tibia izquierda y del peroné derecho. En el fémur derecho se observó, sobre el sector medio-distal de la diáfisis, una reacción osteítica. En toda la tibia derecha y porción 
Tabla 3. Porcentajes de lesiones paleopatológicas por entierro y por sectores del esqueleto: axial y apendicular. Nota: $\mathbf{n}=$ cantidad; prop.= proporción; apen.=apendicular.

\begin{tabular}{|c|c|c|c|c|c|c|c|c|c|c|c|c|c|c|c|c|c|c|}
\hline \multirow{2}{*}{ Entierros } & \multirow{2}{*}{$\begin{array}{l}\text { № huesos } \\
\text { adultos }\end{array}$} & \multicolumn{2}{|c|}{ Patología } & \multicolumn{5}{|c|}{ Degenerativo } & \multicolumn{5}{|c|}{ Traumático } & \multicolumn{5}{|c|}{ Infeccioso } \\
\hline & & $\mathrm{n}$ & $\%$ & $\% \mid \%$ & \%axia & prop. & \%apen. & prop. & $\% 9$ & \%axia & prop. & \%ape & prop. & $\% 9$ & \%axia & rop. & \%aper & prop \\
\hline LP1 & 96 & 42 & 44 & 98 & 67 & $12 / 18$ & 37 & $29 / 78$ & 2 & 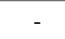 & - & 4 & $1 / 78$ & - & - & - & - & - \\
\hline LP2 & 99 & 42 & 42 & 95 & 83 & $20 / 24$ & 2 & $20 / 75$ & 5 & & & & $2 / 75$ & - & & & - & - \\
\hline LP3 & 92 & 5 & 55 & 96 & 6 & $14 / 21$ & 4 & $35 / 71$ & 4 & & & & $2 / 71$ & & & & - & - \\
\hline LP4 & 42 & 13 & 31 & 46 & 22 & $2 / 9$ & 12 & $4 / 33$ & 8 & 11 & $1 / 9$ & & - & 46 & & - & 18 & $6 / 33$ \\
\hline
\end{tabular}

tercio-distal y medial del peroné izquierdo se relevó osteomielitis (Fig. 4). Finalmente, en el cráneo se registraron signos traumáticos en ambos parietales, en especial en el sector posteromedial. Las reacciones óseas se caracterizan por depresiones, con rasgos porosos en la superficie externa del hueso.

\section{DISCUSIÓN: INTEGRACIÓN DE LOS RESULTADOS Y NUEVO ESTADO DE LA CUESTIÓN PARA EL SITIO LA PETRONA}

Los datos arriba mencionados ofrecen un panorama más completo que los informados en la contribución anterior (Martínez y Figuerero Torres 2000). El NMI calculado para el sitio con anteriori- dad era de seis (véase también Martínez 2010). Sin embargo, los análisis cuali-cuantitativos sobre los cuatro entierros recuperados permitieron contabilizar un total de ocho individuos: siete adultos y un subdaulto (de la categoría etaria infante). A pesar de las modificaciones en el NMI, las categorías de edad y sexo de los individuos adultos han permanecido sin cambios con relación a las originalmente planteadas. Esta nueva situación respecto del NMI tiene implicaciones para la cronología asignada al sitio. Originalmente, LP2 fue clasificado como secundario simple, aunque se propuso que, posiblemente, contuviera también huesos de un individuo infante (Martínez y Figuerero Torres 2000:243-244). Los nuevos análisis no solo confirmaron esta situación,

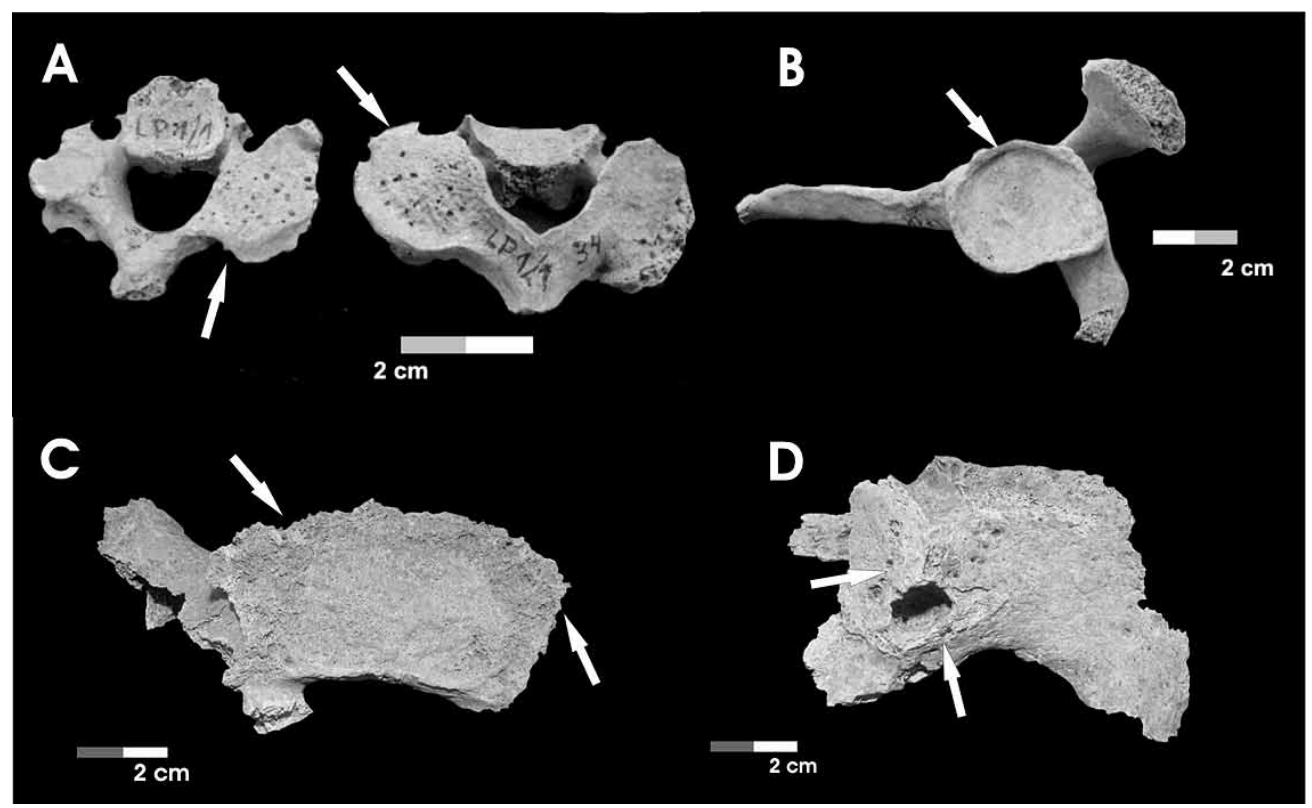

Fig. 3. Lesiones paleopatológicas: A) desgaste óseo, osteofitosis marginal y deformación de las facetas articulares de vértebras cervicales de uno de los individuos de LP1; B) labiación marginal en la cavidad glenoidea de la escápula derecha de uno de los individuos adultos de LP1; C) osteofitosis marginal, deformación de la superficie articular y reacción osteolítica en el anillo cortical del sacro de uno de los individuo adultos de LP2; D) desgaste óseo, reacción osteolítica en el reborde acetabular y desarrollo de absceso en un coxal izquierdo de uno de los individuos adultos de LP2. 


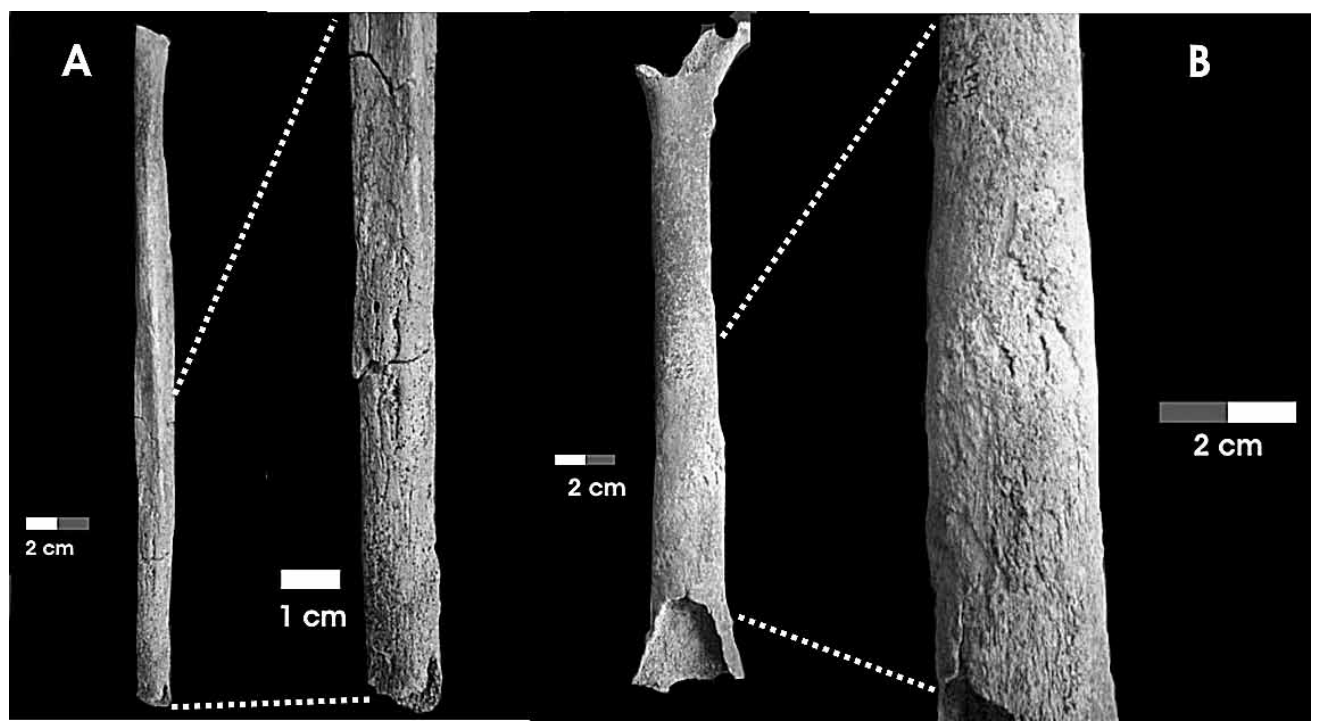

Fig. 4. Infección en huesos del miembro inferior del individuo LP4: A) peroné izquierdo y B) fémur derecho.

sino que se observó la presencia de otro individuo adulto. Los fechados obtenidos para este entierro (ambos realizados sobre costillas de adultos), arrojaron edades de ca. 480 y 770 años AP. Al considerar al entierro como secundario simple y, dado que los demás fechados disponibles para el sitio se localizaban en torno a ca. 500-300 años AP, Martínez (2004:281) consideró al fechado de ca. 770 años AP como posiblemente anómalo. La situación actual respecto del NMI de LP2 (dos adultos y un infante) permite proponer que ambos fechados son válidos y que las costillas datadas provienen de individuos diferentes cuya muerte se produjo en momentos distintos. Asumiendo esto, la cronología asignada al sitio de acuerdo a los eventos de muerte sería de ca. 770-250 años AP.

Las paleopatologías registradas previamente (véase Martínez y Figurero Torres 2000:237) cayeron casi exclusivamente en la categoría de degenerativas. El análisis más exhaustivo aquí presentado muestra que todos los individuos adultos padecieron de algún tipo de lesión ósea, con frecuencias diferentes, afectando gran parte de los huesos recuperados de cada entierro. En líneas generales, en este trabajo, las paleopatologías degenerativas fueron también las predominantes, seguidas por las infecciosas y las traumáticas (Tabla 3).

En todos los individuos las lesiones óseas de carácter degenerativo responden a la osteoartro- sis, afectando tanto las articulaciones diartrodiales como anfiartrodiales. Existen numerosos factores que pueden generar estas anomalías, entre ellos, deficiencias en el metabolismo, en la nutrición, en la vascularización, traumas, etc. (Weiss y Jurmain 2007). Sin embargo, tales disrupciones fisiológicas no fueron relevadas, con lo cual es posible que el estrés mecánico y las actividades físicas llevadas a cabo por los individuos sean los causantes principales de dichas lesiones (Jurmain 1990; Radin 1982; Weiss 2006, entre otros). Esto cobra sentido si se considera la edad de los individuos, ya que la mayor severidad de las alteraciones osteoartrósicas se manifestó en los adultos medios (LP1 y LP2). Teniendo en cuenta la distribución de las anomalías, el esqueleto axial fue el más comprometido, principalmente la columna vertebral. En el caso de las vértebras, las modificaciones óseas pudieron resultar de una extremada presión sobre el cráneo o la región posterior del cuello, que comprimió las articulaciones (e.g., acarreo de peso sobre la región del hombro/cuello, traslado de objetos pesados sobre la cabeza, etc.; Aufderheide y Rodríguez-Martín 1998; Campillo 2001; Lovell 1994; Ortner 2003, entre otros). Respecto de los coxales y las escápulas, estos elementos mostraron modificaciones vinculadas con actividades de locomoción y de transporte de objetos pesados comprometiendo las articulaciones. Un caso llamativo se presentó en un individuo adul- 
to de LP2 a través de la presencia de un absceso remodelado en el coxal izquierdo y en la escápula derecha, indicando la inflamación de los tejidos blandos en las zonas articulares y una alteración del sistema muscular (Campillo 2001). Asimismo, las actividades mecánicas habrían generado las lesiones en los huesos largos de los miembros inferiores, y en los huesos de las manos y los pies (Larsen 1997).

A nivel traumático, se relevaron casos aislados que pudieron corresponder a diversos eventos ocurridos en la vida cotidiana como caídas, golpes, accidentes, etc. (e.g., tibia y falanges en LP1 y LP2, respectivamente). Sin embargo, los rasgos observados en el cráneo del individuo de LP4 pueden responder a severos golpes como producto de accidentes (e.g., caídas) o violencia interpersonal. Numerosos autores sostienen que la mayoría de los politraumatismos observados en los cráneos responden a hechos de violencia tanto de índole doméstica como intergrupal (Flensborg 2011; Gordón 2011; Lambert 1997; Larsen 1997; Ortner 2003; Walker 2001, entre otros). La presencia de pequeños poros alrededor de las lesiones se corresponde con deficiencias vasculares, dada la obstrucción del sistema circulatorio producto de los golpes. El estado remodelado de los poros y las semejanzas en sus características morfológicas estarían indicando que las lesiones se produjeron en un mismo momento y que el individuo habría sobrevivido a algún evento traumático.

La periostitis, osteítis y osteomielitis registrado en LP4 son consideradas infecciones no específicas $y$ pueden ser secundarias a diversas causas como actividades irrigativas de origen traumático, infecciones derivadas de lesiones varicosas, aunque con mayor frecuencia son consecuencia de infecciones piogénicas (Aspillaga et al. 2006; Campillo 2001; Larsen 1997). De acuerdo con Campillo (2001:225), el conjunto de lesiones óseas relevadas en la tibia y el peroné, son típicas de la osteomielitis. Este registro osteopatológico da cuenta de una enfermedad infecciosa sistémica que afectó al individuo en las últimas etapas de su vida, aunque no es posible estimar si fue la causa de su muerte. Tampoco es posible evaluar el agente causante de tal infección dada la diversidad de virus y bacterias que existen, aunque numerosos autores coinciden en que la bacteria Staphylococcus aureus sería la principal responsable (Aufderheide y Rodríguez-Martín 1998; Campillo 2001; Ortner 2003, entre otros).
La información tafonómica generada en Martínez y Figuerero Torres (2000) destaca la ausencia de meteorización, la presencia de fracturas producidas en estado seco del hueso y la intensidad de la acción de raíces. Los resultados presentados en este trabajo están en sintonía con estas tendencias, a las que se suman la existencia de procesos diagenéticos y la ausencia de modificaciones por carnívoros y roedores. Es poco probable que la acción mecánica y química de las raíces haya desintegrado y/o dispersado los huesos faltantes en algunos entierros (e.g., LP3), dado que ciertas unidades anatómicas (e.g., fémur, tibia y huesos de los pies) poseen un importante tamaño y se caracterizan por una alta densidad mineral ósea (Behresmeyer 1978; Galloway et al. 1997; Lyman 1994).

En resumen, la integridad anatómica fue relativamente alta, permitiendo lograr resultados a partir de diversos tipos de análisis (e.g., paleopatológicos, determinación de sexo, estimación de la edad de muerte, etc.). La ausencia de algunas partes esqueletarias así como las características de las trazas antrópicas intencionales indican el procesamiento de cadáveres como parte de una práctica social vinculada al tratamiento de los muertos (véase discusión en Martínez 2010). El estudio tafonómico permitió identificar huellas de corte vinculadas con actividades de descarne y raspado en los entierros LP2 y LP4. Dichas huellas son esperadas para contextos de entierros secundarios, como en el caso de LP2, que pueden implicar el desmembramiento de algunas porciones del esqueleto y la limpieza de la superficie de los huesos que contienen tejido blando adherido. Las huellas antrópicas halladas en LP4 indicarían una etapa de remoción de tejidos blandos previa al entierro primario. En este sentido, no habría existido desarticulación del esqueleto sino sólo la intención de extraer las partes blandas. En el caso del entierro primario LP3 cabe destacar la ausencia de huesos largos de los miembros inferiores y coxales, así como el esternón y las clavículas. Los resultados tafonómicos previamente discutidos indican que esta ausencia no puede ser explicada por factores postdepositacionales. Esto refuerza la propuesta del intenso manejo de cadáveres indicado por las evidencias de huellas de procesamiento antrópico, así como la recuperación de partes esqueletarias de entierros primarios (e.g., LP3) para la confección de secundarios (véase Martínez y Figuerero Torres 
2000:241). Esta intensidad en la manipulación de los cuerpos se observa también en la práctica que involucró mantener espacialmente asociadas partes de una misma unidad anatómica. En este sentido, en el entierro secundario LP1 se observó que dos fragmentos de un mismo hueso (húmero) con fracturas en estado seco, que yacían lado a lado, fueron acomodados intencionalmente (véase Martínez y Figuerero Torres 2000:241). Asimismo, los valores diacrónicos de los fechados obtenidos para LP2 sugieren que los grupos humanos habrían estado recolectando cadáveres de individuos que tuvieron distintos momentos de muerte, posiblemente, procedentes de diferentes sectores del paisaje y cuyas unidades anatómicas habrían sido eventualmente "juntadas", ordenadas y dispuestas en un entierro secundario.

\section{CONCLUSIONES}

Los análisis llevados a cabo en este trabajo permitieron profundizar en los resultados obtenidos en una contribución previa (Martínez y Figuerero Torres 2000). En este sentido, se obtuvo un NMI mayor que, a su vez, llevo a redefinir la cronología, modalidad de entierro y las categorías etarias que se encuentran representadas en los entierros. El estudio paleopatológico más sistemático permitió registrar una mayor cantidad de unidades anatómicas afectadas y una mayor variabilidad de grupos etiológicos (e.g., degenerativas, traumáticas $e$ infecciosas) distribuidos en forma diferencial entre los individuos y a través del tiempo. Los estudios tafonómicos ratificaron las tendencias previamente observadas, agregaron información sobre la ausencia de importantes agentes naturales (e.g., carnívoros y roedores) y pusieron de manifiesto las prácticas antrópicas intencionales. En este sentido, se observó una intensa manipulación de cadáveres en ciertos sectores del paisaje durante un lapso prolongado de tiempo (770-250 años AP). Simultáneamente, junto a estas prácticas mortuorias, el sitio fue intensamente reocupado y utilizado como un lugar de actividades múltiples, domésticas, a juzgar por la cultura material hallada en el médano (véase discusión en Martínez 2008-2009, 2010). La evidencia discutida permite proponer que en este lugar del paisaje se solaparon actividades domésticas y sagradas a través del tiempo (Martínez 2010).
El sitio La Petrona puede considerase como un lugar persistente (sensu Schlanger 1992:92), teniendo en cuenta el uso repetitivo del lugar a través del tiempo y para diversos fines (e.g., explotación de recursos, acceso al agua, practicas sagradas vinculadas con el manejo de cadáveres, etc.). El retorno a este lugar habría estado mediado tanto por las características del entorno ambiental como social (Goldstein 1995; Litteton 2007; Litteton y Allen 2007; Pardoe 1988). Este trabajo permitió aportar evidencias e interpretaciones novedosas para el sitio y el área de estudio que serán útiles para futuras discusiones referidas al manejo de cuerpos humanos, prácticas mortuorias y funcionalidad de sitios.

\section{AGRADECIMIENTOS}

Este trabajo fue posible gracias a los subsidios otorgados por el CONICET (PIP-CONICET № 6147/05) y ANPCyT (PICT № 264). Al INCUAPA por la disponibilidad del laboratorio y el gabinete. A la Lic. Luciana Stoessel y al Dr. Pablo Messineo por los valiosos comentarios realizados al trabajo. A los evaluadores por sus comentarios y sugerencias.

\section{BIBLIOGRAFÍA}

ASPILLAGA, E., M.D. CASTRO, M. RODRÍGUEZ y C. OCAMPO 2006. Paleopatología y estilo de vida: El ejemplo de Los Chonos. Magallania 34(1): 77-85.

AUFDERHEIDE, A.C. y C. RODRÍGUEZ-MARTíN 1998. The Cambridge Enciclopedia of Human Paleopathology. Cambridge University Press, Cambridge.

BASS, W.M. 1995. Human Osteology: A Laboratory and Field Manual of the Human Skeleton. Special Publication, Missouri Archaeological Society. Columbia.

BAYALA, P. 2010. El registro bioarqueológico del sitio Paso Alsina 1 (curso inferior del río Colorado, provincia de Buenos Aires). Estudio de la estructura sexual y etaria de cuatro entierros secundarios. En Mamul Mapü: Pasado y presente desde la arqueología aampeana, editado por M. Berón, L. Luna, M. Bonomo, C. Montalvo, C. Aranda y M. Carrera Aizpitarte, pp. 123-136, Tomo I. Libros del Espinillo, Ayacucho, Buenos Aires.

BAYÓN, C., G. MARTÍNEZ, G. ARMENTANO y C. SCABUZZO 2004. Arqueología del valle inferior del río Colorado: el sitio La Primavera. Intersecciones en Antropología 5: 39-53. 
BEHRENSMEYER, A.K. 1978. Taphonomic and ecological information from bone weathering. Paleobiology 4(2): 150-162.

BINFORD, L. 1981. Bones: Ancient Men and Modern Myths. Academic Press. New York.

BLUMENSCHINE, R.J., C.W. MAREAN y S.D. CAPALDO 1996. Blind tests of interanalyst correspondence and accuracy in the identification of cut marks, percussion marks, and carnivore tooth marks on bone surfaces. Journal of Archaeological Science 23: 493-507.

BOCEK, B. 1986. Rodent ecology and burrowing behavior: Predicted effects on archaeological site formation. American Antiquity 51(3): 589-603.

BROOKS, S. y J.M. SUCHEY 1990. Skeletal age determination based on the os pubis: A comparison of the Acsádi Nemeskéri and Suchey Brooks methods. Human Evolution 5(3): 227238.

BUIKSTRA, J. y D. UBELAKER 1994. Standards for data collection from human skeletal remains. Archaeological Survey Research Series 44. Arkansas.

CAMPILLO, D. 2001. Introducción a la Paleopatología. Edición Ballaterra S. L., Barcelona.

FISHER, J.W. 1995. Bone surface modifications in zooarchaeology. Journal of Archaeological Method and Theory 2: 7-68.

FLENSBORG, G. 2010. Análisis paleopatológicos en el sitio Paso Alsina 1. Primeros resultados sobre la salud de las sociedades cazadoras-recolectoras en el valle inferior del río Colorado durante el Holoceno tardío final. En Mamül Mapu: Pasado y presente desde la arqueología pampeana, editado por M. Berón, L. Luna, M. Bonomo, C. Moltalvo, C. Aranda y M. Carrera Aizpitarte, pp. 165-180, Tomo I. Editorial Libros del Espinillo, Ayacucho, Buenos Aires.

FLENSBORG, G. 2011. Lesiones traumáticas en cráneos del sitio Paso Alsina 1. Explorando indicadores de violencia interpersonal en la transición Pampeano-Patagónica Oriental (Argentina). Intersecciones en Antropología, en prensa.

GALLOWAY, A., P. WILLEY y L. SNYDER 1997. Human bone mineral densities and survival of bone elements: A contemporary sample. En Forensic Taphonomy: The posmortem fate of human remains, editado por W.D. Haglund and M.H. Sorg, pp. 295-317. CRC Press, New York.

GILBERT, R. y T.W. MCKERN 1973. A method for ageing the female of pubis. American Journal of Physical Anthropology 23: 149-164.

GOLDSTEIN, L.G. 1995. Landscapes and mortuary practices. A case for regional perspectives. En Regional Appro- aches to Mortuary Analysis, editado por L.A. Beck, pp 101-121. Plenum Press, New York.

GONZALEZ, M. 2010. Huellas de corte y análisis contextual en restos óseos humanos de la cuenca inferior del río Colorado: implicaciones para el entendimiento de las prácticas mortuorias. En Mamul Mapü: pasado y presente. Perspectivas de la arqueología pampeana a comienzos del tercer milenio, editado por M. Berón, L. Luna, M. Bonomo, C. Moltalvo, C. Aranda y M. Carrera Aizpitarte, pp. 193-210, Tomo I. Editorial Libros del Espinillo, Ayacucho, Buenos Aires.

GORDÓN, F. 2011. Dinámica poblacional, conflicto y violencia en el norte de Patagonia durante el Holoceno Tardío: un estudio arqueológico. Tesis Doctoral inédita. Facultad de Ciencias Naturales y Museo, Universidad Nacional de La Plata, La Plata.

JURMAIN, R.D. 1990. Paleoepidemiology of a central California prehistoric population from CA-ALA-329: II. Degenerative disease. American Journal of Physical Anthropology 83: 83-94.

JURMAIN, R.D. 1991. Paleoepidemiology of trauma in a prehistoric central California population. En Human Paleopathology: Current Syntheses and Future Options, editado por D.J. Ortner y A.C. Aufderheide, pp. 241-248. Smithsorian Institution Press. Washington.

LAMBERT, P. 1997. Patterns of violence in prehistoric huntergatherers societies of coastal southern California. En Troubled Times: Violence and Warfare in the Past, War and Society, editado por D.L. Martin y D.W. Frayer, pp. 77-109. Gordon and Breach Publishers, Amsterdam.

LARSEN C.S. 1997. Bioarchaeology. Interpreting Behavior from the Human Skeleton. Cambridge University Press, Cambridge.

LITTLETON, J. 2007. From the perspective of time: huntergatherer burials in south-eastern Australia. Antiquity 81: 1013-1081.

LITTLETON, J. y H. ALLEN 2007. Hunter-gatherer burial and creation of persistent places in southeastern Australia. Journal of Anthropological Archaeology 26: 283-298.

LOWELL, N.C. 1994. Spinal arthritis and physical stress at Bronze Age Harappa. American Journal of Physical Anthropology 93: 149-164.

LOVEJOY, O., R. MEINDL, T. PRYZBECK y R. MENSFORTH 1985. Chronological methamorphosis of the auricular surface of the ilium: a new method for the determination of adult skeletal age at death. American Journal of Physical Anthropology 68: 15-28.

LYMAN, R.L. 1994. Vertebrate Taphonomy. Cambridge University Press, Cambridge. 
MARTÍNEZ, G. 2004. Resultados preliminares de las investigaciones arqueológicas realizadas en el curso inferior del río Colorado (partidos de Villarino y Patagones, provincia de Buenos Aires). En Aproximaciones contemporáneas a la arqueología pampeana. Perspectivas teóricas, metodológicas, analíticas y casos de estudio, editado por M.A. Gutierrez G. Martínez, R. Curtoni, M. Berón y P. Madrid, pp. 275-292. Facultad de Ciencias Sociales, Universidad Nacional del Centro de la Provincia de Buenos Aires, Olavarría.

MARTÍNEZ, G. 2008-2009. Arqueología del curso inferior del río Colorado: estado actual del conocimiento e implicaciones para la dinámica poblacional de cazadores-recolectores pampeanos-patagónicos. En Cazadores-Recolectores del Cono Sur. Revista de Arqueología 3, editado por D.L. Mazzanti, M.A. Berón y F. W. Oliva, pp. 73-94. Editorial Universitaria de Mar del Plata, Mar del Plata.

MARTÍNEZ, G. 2010. Entierros humanos en lugares sagrados y domésticos durante el Holoceno tardío: El registro bioarqueológico del curso inferior del río Colorado (Provincia de Buenos Aires, Argentina). Revista Werken 13: $145-160$.

MARTÍNEZ, G. y M.J. FIGUERERO TORRES 2000. Sitio arqueológico La Petrona (partido de Villarino, Provincia de Buenos Aires): Análisis de las modalidades de entierro en el área sur pampeana. Relaciones de la Sociedad Argentina de Antropología XXV: 227-247.

MARTÍNEZ, G., G. FLENSBORG, P. BAYALA y R. LÓPEZ 2007. Análisis de la composición anatómica, sexo y edad de dos entierros secundarios del sitio Paso Alsina 1 (Pdo. de Patagones, Pcia. de Buenos Aires). En Arqueología en Las Pampas, editado por C. Bayón, A. Pupio, Ma. I. González, N. Flegenheimer y M. Frére, pp. 41-58. Vol. I. Sociedad Argentina de Antropología, Buenos Aires.

MARTÍNEZ, G., A.F. ZANGRANDO y L. PRATES 2009. Isotopic ecologic and human palaeodiets in the lower basin of the Colorado River, Buenos Aires province, Argentina. International Journal of Osteoarchaeology 19: 281-296.
ORTNER, D.J. 2003. Identification of Pathological Conditions in Human Skeletal Remains. Academic Press, New York.

PARDOE, C. 1988. The cemetery as symbol. The distribution of prehistoric aboriginal burial grounds in southeastern Australia. Archaeology in Oceania 23: 1-16.

POWELL, M.L. 1988. Status and health in prehistory: A case of study of the Moundville Chiefdom. Smithsonian Institution Press, Washington D.C.

PRATES, L., G. MARTÍNEZ y C. SCABUZZO 2006. Evidencias arqueológicas del Holoceno tardío final en el curso medio del río Colorado (Provincia de Río Negro). Sitio Don Aldo 1. En Cazadores Recolectores del Cono Sur. Revista de Arqueología 1, editado por L. Mazzanti, M.A. Berón y F.W. Oliva, pp. 163-177. Editorial Universitaria de Mar del Plata, Mar del Plata.

RADIN, E.L. 1982. Mechanical factors in the causation of osteoarthritis. Rheumatology 7: 46-52.

REITZ E.J. y E.S. WING 1999. Zooarchaeology. Cambridge Manuals in Archaeology. Cambridge University Press Cambridge.

SCHEUER, L. y S. BLACK 2000. Developmental Juvenile Osteology. Academic Press. London.

SCHLANGER, S. 1992. Recognizing persistent places in Anasazi settlement systems. En Space, Time and Archaeological Landscape, editado por J. Rossignol and L. Wandsnider, pp. 91-112. Plenum Press, New York.

SUCHEY, J.M. y D. KATZ 1986. Applications of pubic age determination in a forensic setting. En: Forensic Osteology: Advances in the Identification of Human Remains, editado por K.J. Reichs, pp. 204-236. Sprigfield, Illinois.

WALKER, P.L. 2001. A bioarchaeological perspective on the history of violence. Annual Review of Anthropology 30: 573-596

WEISS, E. 2006. Osteoarthritis and body mass. Journal of Archaeological Science 33: 690-695.

WEISS, E. y R.D. JURMAIN 2007. Osteoarthritis revisited: A contemporary review of etiology. International Journal of Osteoarchaeology 17: 437-450. 
